\title{
Throughput Analysis for Cooperative Cognitive Radio Networks using Cyclostationary Detection
}

\author{
Enfel Barkat, \\ Department of Electrical Engineering, Effat University, Jeddah Kingdom of Saudi \\ Arabia
}

Received: July 25, 20, 2020. Revised: November 7, 2020. Accepted: December 1, 2020. Published: December 29, 2020.

\begin{abstract}
Detection of primary users (PUs) in the presence of interference and noise and improvement of spectrum utilization is one of the aims of cognitive radio (CR). In this paper, a fully distributed cooperative spectrum sensing scheme based on cyclostationary features techniques is proposed. The primary signal detection is realized by an orthogonal frequency division multiplexing (OFDM) sensing algorithm; each secondary user (SU) makes decisions about the PU by exchanging their own measurements with the local neighbors. The distributed scheme is analyzed on a network layer, where the throughput performance is analyzed in terms of sensing accuracy, frame duration, and system overhead. An analytical expression for the SU throughput is derived, in addition to investigating the issue of trade offs between time and overhead. Simulation results showed the relationship between the throughput and the sensing time, the effect of increasing the number of SUs on the throughput, and the outcome of increasing traffic intensity on the system performance.
\end{abstract}

Keywords Cognitive radio, spectrum sensing, throughput.

\section{INTRODUCTION}

The mobile communication system is a rapidly growing field to support people's activities. This growth leads to high demand for wireless radio spectrum usage. Traditionally, the radio spectrum scheme is managed for specific use. There is a situation in which some radio spectrums are fully utilized by the users, whereas, others are underutilized.
Obviously, static frequency allocation schemes cannot accommodate high demand of the radio spectrum. CR is an emerging technology proposing the concept of dynamic spectrum access as a solution to the looming problem of spectrum scarcity caused by the rigid spectrum allocation and the underutilization of spectral resources [1], [2]. A CR network is composed of multiple SUs also called nodes which are deployed in the area of observation. Depending on the application and on the coverage area, the network can consist of a reduced number of sensor nodes, or it can be a large-scale deployment composed of hundreds of units. A standard node is usually composed of a transducer in charge of sensing the physical parameters, a radio transceiver for wireless communications, a low complexity processing unit, and a power supply - normally in the form of a battery. The sensors gather measurements from the environment and eventually make simple processing of the sensed data. The data can be transferred to a central node in a centralized network, or it can be locally processed instead in a decentralized network. The connection and topology between the nodes depends on the transmission power and on their geographical locations and the optimization methods to be deployed.

In typical centralized deployments, each node sends its observations to a complex and 
intelligent unit denoted the fusion center (FC). The FC is in charge of collecting data from the network and making the final decision about the presence of the PU. Gathering the entire collected data at one place may be very difficult under communication constraints. Centralized networks require a proper organization of the nodes and the implementation of medium access control (MAC), as well as routing protocols to forward the data to the FC. Communication errors and packet drops can affect the performance of such a network. It is necessary for every node to establish a connection with the FC which leads to a higher overall cost of the network, especially when the number of nodes becomes large. For these reasons, a centralized CR network becomes highly inefficient [3].

Most of the cooperative sensing (CS) schemes stem from the field of distributed detection [4]. Figure 1 shows an example of CS, where $N$ SUs sense listening channels for the PU signal activity and send the sensing information on reporting channels to the FC, which makes the final decision. It is very unlikely that all the channels between the PU and the SUs will be in a deep fade simultaneously. Thus, cooperative detection helps in mitigating the channel effects through multipath diversity [5].

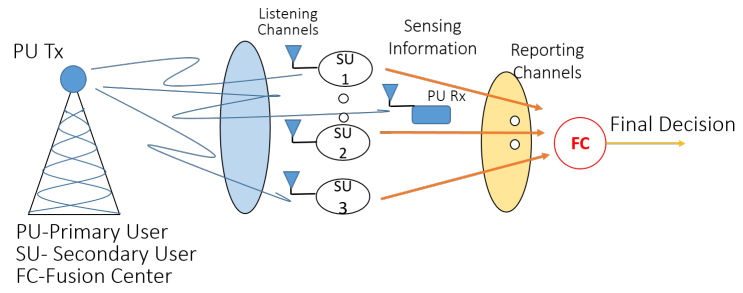

Fig. 1. CS: SUs sense a common PU on the listening channels and send the sensing information to a FC over reporting channels.

In centralized cooperative spectrum sensing, every SU needs to establish a connection with the common receiver which requires the implementation of a network protocol. A relay route to the FC must be implemented for SUs located far away from the common receiver. When a decision is made, there should be a reliable wireless broadcasting channel to inform every user [6].

An alternative is a decentralized network, where each node has the capability of selfconfiguration and self-organization. The nodes change their links to other devices frequently where they establish a dynamic network without the need of a fixed infrastructure. In a decentralized network, a node can function both as a network router for routing packets from other nodes as well as a network host for transmitting and receiving data. This network is expected to provide reliable results approaching a globally optimal solution available at each node. A distributed cooperative spectrum sensing system is a selfconfiguring system where users can establish a dynamic network without the need of a fixed infrastructure. Every user is independent and will change its links to other users frequently [6]. A node can function both as a network router for routing packets from other nodes and as a network host for transmitting and receiving data as shown in Figure 2.

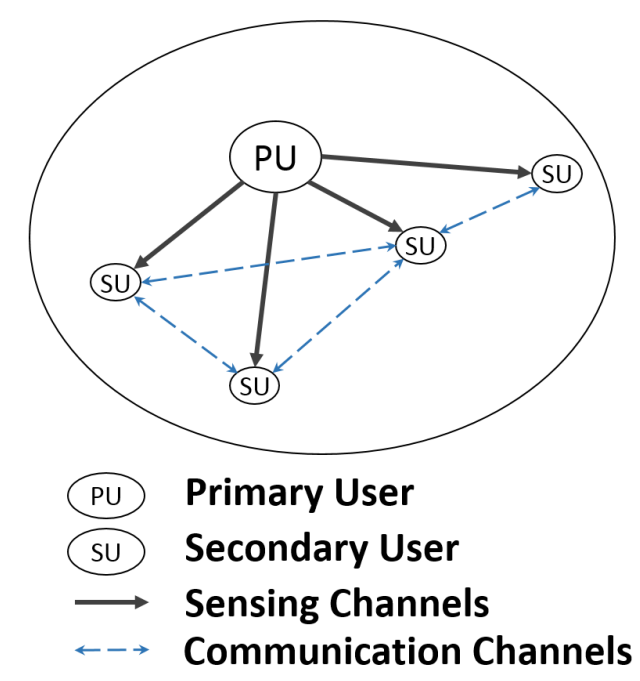

Fig. 2. Distributed cooperative spectrum sensing. 
Figure 2 demonstrates how SUs receive initial information about the PU using sensing channels, after which they use communication channels to make decision about the presence of the PU and chooses the information exchange rate according to the estimated average signal-to-noise ratio (SNR). Finally, SUs exchange their own measurement with their local neighbors to make a decision about the PU. Distributed systems are particularly useful when a reliable fixed infrastructure is not available.

Spectrum sensing is a crucial task whereby the SU senses the spectrum to detect the presence or absence of any PU signals to prevent interference, and identify spectrum opportunity for secondary access [1] [7]. Sensing the spectrum continuously in time is not practical in CR systems, as it would take up all the receiver resources leaving no space to transmit-receive data. Therefore, periodic sensing is considered for sensing the spectrum. For the periodic sensing technique, if the PU is transmitting while the CR user is not sensing, it is not possible for the SU to detect it. cooperative spectrum sensing is used to enhance the sensing performance of each SU. In addition, to improving the detection performance, cooperative spectrum sensing reduces the spectrum sensing time [3].

In this paper, we evaluate the throughput of the SUs and investigate the issue of how to strike desirable trade-offs between sensing time and overhead in a wireless fading network. We study how varying the number of SUs in uncertain environments such as channel fading, effect the data rate and the sensing accuracy of the CR network. Simulation results show that a significant improvement in the throughput of the SUs is achieved when the parameters for the sensing threshold and the throughput are jointly optimized. Finally, we look into the effect of traffic intensity on the throughput.

\section{Throughrut With PERIODIC SPECTRUM SENSING}

The periodic spectrum sensing for a single cognitive node is depicted in Figure 3. The SU periodically senses the spectrum with a period of $T_{s}$ seconds every period. The CR node will detect the PU only when the PU is transmitting while the $\mathrm{CR}$ is sensing the spectrum. Otherwise, the SU switches off its transmission until it discerns the PU is not transmitting [8].



Fig. 3. Arrangement of frames in a CR network

Note: this model works only when the PU is active or absent during the whole SU frame.

The sensing and the communicating channels shown in Figure 2 are intertwined with each other where there is a tradeoff between the spectrum sensing and the SUs throughput. A long sensing time keeps the PU safe from interference since there will be less time for the SU to access the PU band. This causes the SU throughput to be very low. On the other hand, a short sensing time makes the PU more subject to interference and maximizes the throughput of the SU. This can be interpreted in terms of probability of detection and probability of false alarm. As the sensing time increases, the false alarm probability decreases and the probability of detection increases which makes the primary user less exposed to secondary interference. [9]

The sensing-throughput tradeoff has received a lot of interest from researchers. In [9], Liang et al. studied and mathematically formulated the problem of designing the sensing duration 
to maximize the throughput for the SU when sensing and communicating channels are subjected to AWGN. They proved via simulation that there is an optimal sensing time which yields to the highest throughput for the secondary network. In [10], the authors studied the sensing-throughput tradeoff problem using double thresholds over AWGN channels. Their iterative algorithm showed a significant improvement in the throughput for the SUs when the parameters for the number of sensors and the sensing threshold are jointly optimized, In [11], the impact of sensing time and power adaptation on the performance of the CR system, when there exists a Rayleigh flat-fading channel, is considered. The authors assumed AWGN for the sensing channel and proved that sensing time plays an important role in maximizing the system throughput. In [12] and [13], the sensing-throughput tradeoff was investigated for sensing-based spectrum sharing over AWGN channels. They designed the optimal sensing length that maximizes the throughput of a wideband opportunistic CR system.

\section{System Model}

We consider a non-centralized fully distributed CR scheme. Consider again Figure 3 which shows the frame structure for a CR network with periodic spectrum sensing where each frame consists of one sensing slot and one data transmission slot. Suppose the sensing duration is $T_{S}$ and the frame duration is $T_{F}$. The $\mathrm{SU}$ will only transmit if there is no PU active and waits until the next frame to sense again. The transmission channel has the following conditions:

- All transmitted packets have identical lengths each requiring the length of a single slot, of length $T_{f}-T_{s}$ for transmission.

- The transmission by all users is synchronous, where they are allowed to start transmission only at the beginning of some slot; and ideally there are no propagation delays in the channel feedback information obtained by the users.

- If at least two packets attempt transmission within the same slot, a collision occurs and such an event is initially the only cause for faulty transmissions; that is, a slot occupied with a single packet results in successful transmission, while a collision results in complete loss of the information carried by the collided packets. Thus, retransmission of collided packets is then necessary.

- The outcome per slot, possibly accessible by the users named feedback level is either binary, distinguishing between collision versus non-collision, or ternary, distinguishing between collision, versus emptiness versus success. We note that an non-collision event corresponds to a slot that is either empty or occupied with a single packet transmission, while a successful event corresponds to a slot occupied with a single packet whose transmission is then successful.

- Even though the identity of the collided packets is completely lost, the number of packets involved in the collision is revealed and broadcasted to all users.

The data transmission of the SU is activated subject to the spectrum sensing results based on the following hypotheses for each channel [14].

$$
\begin{aligned}
& H_{1}: y(n)=h(n) s(n)+w(n) \\
& H_{0}: y(n)=w(n)
\end{aligned}
$$

where $y(n)=\left[y_{1}\left(n, \ldots, Y_{M}(N)\right)\right]^{T}$ is the observed complex time series containing $M$ samples received at instant $n$. $w(n)$ represents a WGN process with variance $\sigma_{w}^{2} . s(n)$ is the signal to be detected and $h(n)$ is the propagation channel between PU and $M$ SUs. $H_{1}$ and $H_{0}$ represent the occupied and the idle spectrum band hypothesis, respectively. The SU transmits only at $H_{0}$ when the data transmission slot is idle, otherwise it keeps silent. SU traffic is 
queued.

For spectrum sensing, we consider a real time environment sensing scheme as shown in Figure 4. Where the spectrum sensing is always imperfect and sensing errors are categorized intro four scenarios as follows [15]:

1) The channel is idle and the SU decides that the channel is idle, a correct detection occurs and the throughput is maximum.

2) The channel is idle and the SU decides that the channel is busy, a false alarm occurs and the SU does not transmit.

3) The channel is busy and the SU decides that the channel is busy, a correct detection occurs and the SU will not transmit.

4) The channel is busy and the SU decides that the channel is idle, a miss detection occurs and the SU will transmit.

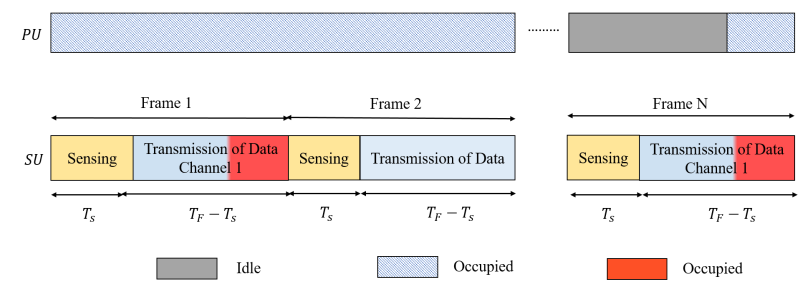

Fig. 4. Data packet transmission for imperfect sensing.

\section{A. Throughput of the SU Under Imperfect Sensing}

We denote $C_{0}$ and $C_{1}$ as the throughput of the secondary network when it operates in the absence of the PU and when it operates in the presence of the PU, respectively [16]. We also denote $P_{p}$ to be the interference power of the PU measured at the secondary receiver, $\mathrm{SNR}_{s}=P_{s} / N_{0}$, where $P_{s}$ is the received power of the $\mathrm{SU}$ and $N_{0}$ is the noise power and $\mathrm{SNR}_{p}=P_{p} / N_{0}$. Both the PU and the SU signals modeled as Gaussian and independent from each other, thus we can write

$$
C_{0}=\log _{2}\left(1+\mathrm{SNR}_{S}\right)
$$

and

$$
C_{1}=\log _{2}\left(1+\frac{P_{s}}{P_{p}+N_{0}}\right)=\log _{2}\left(1+\frac{\mathrm{SNR}_{s}}{1+\mathrm{SNR}_{p}}\right)
$$

For the first scenario (correct detection) and fourth scenario (missed detection) the throughput depending on the sensing time duration $T_{S}$ is given by

$$
B_{0}\left(T_{S}\right)=\frac{T_{F}-T_{S}}{T_{F}} C_{0}
$$

and

$$
B_{1}\left(T_{S}\right)=\frac{T_{F}-T_{S}}{T_{F}} C_{1},
$$

From [9] the total throughput of the SU is

$$
R\left(T_{s}\right)=R_{0}\left(T_{s}\right)+R_{0}\left(T_{s}\right)
$$

where

$$
R_{0}\left(T_{s}\right)=\frac{T_{F}-T_{s}}{T_{F}} C_{0} \cdot\left[1-P_{f}\left(T_{s}\right)\right] \cdot P_{r}\left(H_{0}\right)
$$

and

$$
R_{1}\left(T_{s}\right)=\frac{T_{F}-T_{s}}{T_{F}} C_{1} \cdot\left[1-P_{d}\left(T_{s}\right)\right] \cdot P_{r}\left(H_{1}\right)
$$

The probability of the channel when it is idle, $P_{r}\left(H_{0}\right)$, and the probability of the channel when it is busy $P_{r}\left(H_{1}\right)$, are derived in [17] as

$$
P_{r}\left(H_{0}\right)=\frac{1}{\sqrt{2 \pi \sigma_{0}}} \exp \left(-\frac{\left(\hat{R}_{i}\right)^{2}}{2 \sigma_{0}^{2}}\right)
$$

and

$$
P_{r}\left(H_{1}\right)=\frac{1}{\sqrt{2 \pi \sigma_{0}}} \exp \left(-\frac{\left(\hat{R}_{i}-\sigma_{s}^{2}\right)^{2}}{2 \sigma_{0}^{2}}\right)
$$

$\hat{R}_{i}$ is repeated for convenience to the reader and derived in the appendix

By substituting (10) and (11) into equation (7), the total throughput of the SU becomes 
investment.

$$
\begin{aligned}
R\left(T_{s}\right)= & B_{0}\left[1-P_{f}\left(T_{s}\right)\right] \frac{1}{\sqrt{2 \pi \sigma_{0}}} \exp \left(-\frac{\left(\hat{R}_{i}\right)^{2}}{2 \sigma_{0}^{2}}\right) \\
& +B_{1}\left[1-P_{d}\left(T_{s}\right)\right] \frac{1}{\sqrt{2 \pi \sigma_{0}}} \exp \left(-\frac{\left(\hat{R}_{i}-\sigma_{s}^{2}\right)^{2}}{2 \sigma_{0}^{2}},\right.
\end{aligned}
$$

where

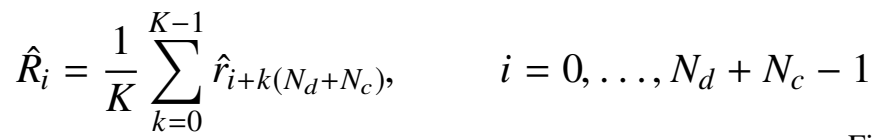

\section{Simulation And Results}

We evaluate the performance of the throughput using the distributed cooperative based spectrum sensing in a Rayleigh fading environment. $N_{c}=8, N_{d}=32$ and $N=100\left(N_{c}+N_{d}\right)$. $P_{r}\left(H_{0}\right)=0.7$ and $P_{r}\left(H_{1}\right)=0.3$. The SNR of the SU $S N R_{S}=20 d B$ and The SNR of the $\mathrm{PU} S N R_{S}=-15 \mathrm{~dB}$. The frame length $T_{F}$ is $20 \mathrm{~ms}$. Probability of detection is chosen to be close but less than 1, especially for low SNR regimes. In IEEE802.22 WRAN $P_{d}$ is chosen to be 0.9 .

\section{A. Throughput vs Sensing Time For Different Number of SUs}

We conduct a Monte Carlo simulation to study the behavior of the throughput as a function of time. The simulation if done for one SU, 5 SUs then 10 SUs. As shown in Figure 5. we can see that there is a trade-off between sensing time and throughput. The throughput in the case of no cooperative sensing is much smaller than in the case of cooperative sensing. Even the minimum throughput with cooperative sensing is achieved with a much longer sensing time and no cooperative sensing. Hence, cooperative sensing with its requirement for information exchange overhead, is more efficient in a fading environment. Hence, there is respectable return on the overhead
Fig. 5. Throughput as a function of sensing time for different number of SUs.

\section{B. Throughput vs Sensing Time For Different SNR Values}

We simulated the throughput as a function of sensing time for different SNR values as illustrated in Figure 6. We can see that throughput improves with the increase of SNR. We also see that if SNR is low, it is better to use more SUs. That is because cooperative spectrum sensing needs more collaborative users to decrease the false alarm probability.

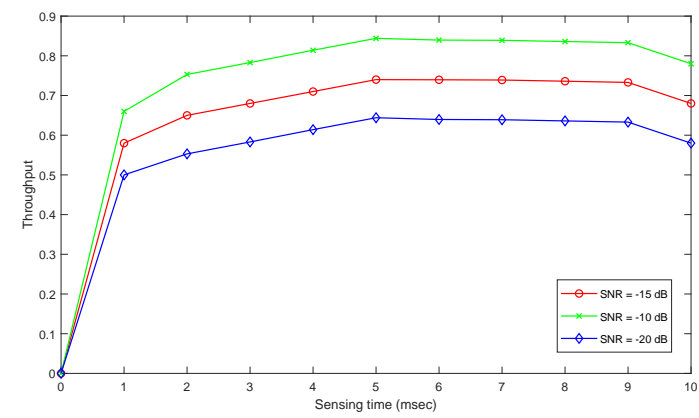

Fig. 6. Throughput as a function of sensing time for different SNR values.

\section{Throughput vs Number of SUs}

Figure 7 shows the throughput as a function of number of SUs for different sensing times. As expected, there is a trade-off between number of SUs and throughput, because increasing SUs reduces the probability of false alarm, but at 
the same time occupies a larger fraction of the frame (more overhead). Therefore, it is observed that there is an optimum point in each curve. It can be seen that the selecting all SUs might not help in achieving the highest throughput. However, it can be achieved through optimizing the number of cooperating SUs. Hence we optimize the number of cooperating SU to improve throughput and reduce network overhead.



Fig. 7. Throughput as a function of number of SUs for different sensing times.

\section{Throughput vs Traffic Intensity For Differ-} ent Number of SUs

In this subsection, simulation results of the throughput for a different number of SUs under heavy traffic is presented in Figure 8. The traffic intensity is the probability that the channel is busy $P_{r}\left(H_{1}\right)$. The probability of packet collisions is higher in a heavy traffic load because more frequent packets transmissions lead to more collisions. In other words, since the primary user has the priority in using the channel and its traffic load is fixed, it happens with a higher probability of becoming active during the SU users transmission.

We can conclude that in high traffic CR networks, the probability of the PU channel been occupied by the PU and SUs is high. This will lead to a high probability of sensing busy and further results in a decrease of throughput. We can also see that using cooperative sensing and increasing the number of SU helps by improving the throughput in high traffic intensity networks.

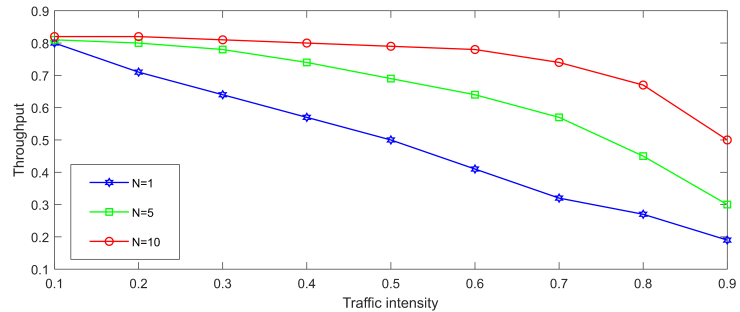

Fig. 8. Throughput vs traffic intensity for various number of SUs.

\section{COnClusion}

we analyzed the throughput for cooperative spectrum sensing on sensing accuracy, frame duration, and system overhead. We considered non-centralized distributed SUs, where the throughput is calculated at each SU separately, then the information is communicated between all the other SUs. We analytically derived and expression for the throughput when the detection is performed using the OFDM cyclostationary detection scheme. Simulation of the SU throughput showed that the relationship between the throughput and the sensing time are inversely proportional. Since a long sensing time keeps the PU safe from interference and gives less time for the SU to access the PU band. We have also concluded that using cooperative spectrum sensing instead of a single user spectrum sensing improves the throughput significantly. Even though one major drawback of cooperative sensing on throughput is the increase in the overhead; it is still in a fading environment. We have also remarked that there is a tradeoff between the number of SUs and throughput, because more SUs means we occupy a larger fraction of the frame. As a result, to achieve the highest throughput we have to find the optimum number of cooperative SUs.

\section{REFERENCES}

[1] S. Haykin et al., "Cognitive radio: brain-empowered wireless communications," IEEE journal on selected areas in communications, vol. 23, no. 2, pp. 201-220, 2005. 
[2] J. Mitola, "Cognitive radio for flexible mobile multimedia communications," in Mobile Multimedia Communications, 1999.(MoMuC'99) 1999 IEEE International Workshop on. IEEE, 1999, pp. 3-10.

[3] T. Yucek and H. Arslan, "A survey of spectrum sensing algorithms for cognitive radio applications," IEEE communications surveys \& tutorials, vol. 11, no. 1, pp. 116130, 2009.

[4] S. H. Sohn, N. Han, J. M. Kim, and J. W. Kim, "Ofdm signal sensing method based on cyclostationary detection," in Cognitive Radio Oriented Wireless Networks and Communications, 2007. CrownCom 2007. 2nd International Conference on. IEEE, 2007, pp. 63-68.

[5] J. Lundén, V. Koivunen, A. Huttunen, and H. V. Poor, "Collaborative cyclostationary spectrum sensing for cognitive radio systems," IEEE Transactions on Signal Processing, vol. 57, no. 11, pp. 4182-4195, 2009.

[6] M. Hasegawa, H. Hirai, K. Nagano, H. Harada, and K. Aihara, "Optimization for centralized and decentralized cognitive radio networks," Proceedings of the IEEE, vol. 102, no. 4, pp. 574-584, 2014.

[7] E. Hossain and V. K. Bhargava, Cognitive wireless communication networks. Springer Science \& Business Media, 2007.

[8] K. Sithamparanathan and A. Giorgetti, Cognitive radio techniques: spectrum sensing, interference mitigation, and localization. Artech House, 2012.

[9] Y.-C. Liang, Y. Zeng, E. C. Peh, and A. T. Hoang, "Sensing-throughput tradeoff for cognitive radio networks," IEEE transactions on Wireless Communications, vol. 7, no. 4, pp. 1326-1337, 2008.

[10] J. Jafarian and K. A. Hamdi, "Non-cooperative doublethreshold sensing scheme: A sensing-throughput tradeoff," in 2013 IEEE Wireless Communications and Networking Conference (WCNC). IEEE, 2013, pp. 33763381.

[11] K. Hamdi and K. B. Letaief, "Power, sensing time, and throughput tradeoffs in cognitive radio systems: A crosslayer approach," in 2009 IEEE Wireless Communications and Networking Conference. IEEE, 2009, pp. 1-5.

[12] X. Kang, Y.-C. Liang, H. K. Garg, and L. Zhang, "Sensing-based spectrum sharing in cognitive radio networks," IEEE Transactions on Vehicular Technology, vol. 58, no. 8, pp. 4649-4654, 2009.

[13] S. Stotas and A. Nallanathan, "On the outage capacity of sensing-enhanced spectrum sharing cognitive radio systems in fading channels," IEEE Transactions on communications, vol. 59, no. 10, pp. 2871-2882, 2011.

[14] W. Tang, M. Z. Shakir, M. A. Imran, R. Tafazolli, and M.-S. Alouini, "Throughput analysis for cognitive radio networks with multiple primary users and imperfect spectrum sensing," IET communications, vol. 6, no. 17, pp. 2787-2795, 2012.

[15] J. Yang and H. Zhao, "Enhanced throughput of cognitive radio networks by imperfect spectrum prediction," IEEE Communications Letters, vol. 19, no. 10, pp. 1738-1741, 2015.

[16] W. Khalid and H. Yu, "Optimal sensing performance for cooperative and non-cooperative cognitive radio networks," International Journal of Distributed Sensor Networks, vol. 13, no. 11, p. 1550147717744992, 2017.
[17] K. Berbra, M. Barkat, and A. Anou, "Pn code acquisition using smart antenna and adaptive thresholding cfar based on ordered data variability for cdma communications," Progress In Electromagnetics Research, vol. 57, pp. 139$155,2014$.

[18] M. K. Simon and M.-S. Alouini, Digital communication over fading channels. John Wiley \& Sons, 2005, vol. 95.

[19] V. P. Ipatov, Spread spectrum and CDMA: principles and applications. John Wiley \& Sons, 2005.

[20] R. Schafer, "Discrete-time signal processing," 1989.

[21] M. Barkat and P. K. Varshney, "Decentralized cfar signal detection," IEEE Transactions on Aerospace and Electronic Systems, vol. 25, no. 2, pp. 141-149, 1989.

[22] T. E. Bogale and L. Vandendorpe, "Multi-cycle cyclostationary based spectrum sensing algorithm for ofdm signals with noise uncertainty in cognitive radio networks," in Military Communications Conference, 2012-MILCOM 2012. IEEE, 2012, pp. 1-6.

\section{APPENDIX}

The primary signal is modeled using OFDM based system with $N_{d}$ subcarriers. The sum of subcarriers representing the OFDM signal are modulated using quadrature amplitude modulation (QAM) [18]. Then, one OFDM symbol of $N_{d}$ duration is obtained by implementing inverse discrete Fourier transform (IDFT) to the g $N_{d}$ complex symbols $d_{i}, i=0, \ldots, N_{d-1}$. [17]. Furthermore, to eliminate the effect of Inter Symbol Interference (ISI) almost completely, a guard time of length $N_{c}$ is introduced in each OFDM symbol. The guard time is chosen larger than the maximum delay spread such that multiplicity components from ne symbol cannot interfere with the next symbol. The new sequence of duration $N_{s}=N_{c}+N_{d}$ samples is expressed as:

$s=\left[s\left(-N_{c}\right) s\left(-N_{c}+1\right) \cdots s(0) s(1) \cdots s\left(N_{d}-1\right)\right]^{T}$

where $T$ denotes transpose. Usually an OFDM frame contains several such blocks. The entire endless serial stream is transmitted over the wireless channel to the receiver. Let $\theta$ be the time offset, i.e. the delay after which the SU receiver receives the first OFDM symbol. If we consider that the received signal contains $K$ OFDM symbols, we use an observation window of length $N$ samples $N=K\left(N_{d}+\right.$ 
$\left.N_{c}\right)+N_{d}$ as illustrated in Fig. 9 In an additive white Gaussian noise (AWGN) channel, the secondary user signal can be written as:

$$
y(k)=s(n)+n(k), k=0,1, \cdots, K\left(N_{d}+N_{c}\right)+N_{d}-1
$$

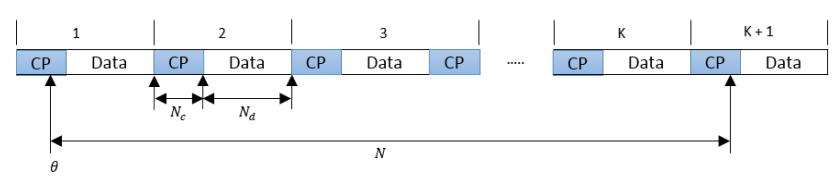

Fig. 9. Model of $N$-samples of received OFDM signal.

The fundamental problem of spectrum sensing is to reliably detect the presence of absence of the primary signal, then decide about spectrum availability [19] [20]. The formulated binary hypothesis test is as follows [21]:

$$
\left\{\begin{array}{l}
H_{1}: y(k)=s(n)+n(k) \\
H_{0}: y(k)=n(k), k=0,1, \cdots, K\left(N_{d}+N_{c}\right)+N_{d}-1
\end{array}\right.
$$

where $H_{1}$ and $H_{0}$ correspond to the presence and absence of an OFDM signal, respectively. The number of samples during a sensing period is denoted as $L=K\left(N_{d}+N_{c}\right)+N_{d}$ and $n(k)$ is the additive white Gaussian [22]. The measure of correlation between two OFDM symbols is described as

$$
\hat{R}_{i}=\frac{1}{K} \sum_{k=0}^{K-1} \hat{r}_{i+k\left(N_{d}+N_{c}\right)}, i=0, \ldots, N_{d}+N_{c}-1
$$

\section{Creative Commons Attribution License 4.0 (Attribution 4.0 International, CC BY 4.0)}

This article is published under the terms of the Creative Commons Attribution License 4.0

https://creativecommons.org/licenses/by/4.0/deed.en_US 